\title{
COMPARACIÓN DE LA GESTIÓN DEL RIEGO AUTOMATIZADO CON TENSIOMETROS FRENTE A LA ESTIMACIÓN DE LA EVAPOTRANSPIRACIÓN EN CULTIVOS HORTICOLAS BAJO INVERNADERO
}

\author{
Alonso López, F., Contreras París, J.I., Baeza Cano, R.
}

Instituto de Investigación y Formación Agraria y Pesquera de Andalucía (IFAPA), Centro La Mojonera, Camino San Nicolás, n¹, 04745 La Mojonera, Almería.

rafaelj.baeza@juntadeandalucia.es

\section{Resumen}

La sostenibilidad del uso del agua en la agricultura se ha convertido en una prioridad y la adopción de estrategias de riego que permitan un uso eficiente de la misma manteniendo niveles de producción satisfactorios, en una necesidad. En particular, en áreas que presentan escasez de recursos hídricos, como la Mediterránea, resulta imprescindible maximizar la eficiencia en el uso del agua y la productividad de la misma. En este trabajo se compara la gestión del riego automatizado empleando tensiómetros electrónicos con la estimación de las necesidades hídricas en un cultivo de calabacín en invernadero. La comparación entre los consumos de agua de los tres tratamientos de riego ensayados y la $\mathrm{ET}_{\mathrm{c}}$ estimada a partir de datos reales de clima $\left(\mathrm{ET}_{\mathrm{c}-\mathrm{r}}\right)$ muestra que el tratamiento $\mathrm{T} 2$ con una consigna de potencial matricial de $-25 \mathrm{kPa}$ y una dotación de $2 \mathrm{~L} \mathrm{~m}^{-2}$ tuvo un consumo igual al calculado mediante la $\mathrm{ET}_{\mathrm{c}-\mathrm{r}}\left(315 \mathrm{Lm}^{-2}\right)$. Este tratamiento junto con el T3 $\left(-40 \mathrm{kPa}\right.$ y $\left.3 \mathrm{~L} \mathrm{~m}^{-2}\right)$ presentaron los mejores resultados en cuanto a eficiencia en el uso del agua. Sin embargo, el T3 redujo significativamente la cosecha en un 16\%. Por tanto, la activación automática del riego con la consigna del T2 fue la que mejores resultados arrojó aunando eficiencia y productividad.

\section{1- Introducción y objetivos}

El uso eficiente del agua se ha convertido en uno de los mayores desafíos mundiales $y$, la agricultura, en este contexto, constituye un factor clave en la mejora de la gestión del agua por ser el principal consumidor de este recurso ( $75 \%$ del consumo total). En particular, en áreas que presentan escasez de recursos hídricos, como la cuenca Mediterránea, resulta imprescindible maximizar la eficiencia en el uso del agua y la productividad de la misma. En este sentido, los cultivos hortícolas en invernadero son un modelo a seguir. La productividad del agua obtenida con este sistema productivo es considerablemente más elevada que la de los cultivos al aire libre (Gallardo et al. 2007). No obstante, todavía existe un margen de mejora que es necesario abordar para asegurar la sostenibilidad del sistema.

La programación del riego permite decidir cuándo regar y qué cantidad aplicar, pero está sujeta a la correcta definición de los parámetros que la definen: dotación y frecuencia. La dotación debe ir asociada al tipo de suelo, considerando textura y estructura, así como profundidad del mismo. La frecuencia irá asociada a las necesidades hídricas del cultivo, $\mathrm{ET}_{\mathrm{C}}$, a lo largo de su ciclo de desarrollo. Su determinación puede estar basada en medidas climáticas, de hidratación de los cultivos o de humedad de suelo. En base a medidas climáticas se ha determinado la $\mathrm{ET}_{\mathrm{c}}$ de la mayoría de los cultivos hortícolas bajo 
invernadero en el sureste peninsular (Fernández et al. 2001). Para optimizar la gestión del riego las investigaciones más recientes se centran en la medida del estado hídrico de la planta o en la medida directa de agua en el suelo (Buttaro et al. 2015, Létourneau et al. 2015). La medida del estado hídrico de la planta todavía presenta muchas dificultades teóricas y prácticas (Jones 2004). Sin embargo, la medida directa del agua en el suelo, ya sea el contenido de agua o el potencial matricial del suelo, es más factible por ser relativamente fácil de medir y automatizar (Van lersel et al. 2013). Los tensiómetros, que determinan el potencial matricial del suelo, se prefieren a menudo frente a otro tipo de sensores de humedad de suelo debido a la precisión y rapidez de la medición, su bajo coste y simplicidad de uso y a que las medidas no están influenciadas por la temperatura o el potencial osmótico del suelo. No menos importante es la posibilidad que ofrecen de adquirir electrónicamente datos a través de transductores de presión diferencial (Thalheimer 2003) permitiendo la automatización de la fertirrigación.

El éxito de la gestión del riego empleando tensiómetros electrónicos para la activación automática del riego depende del establecimiento del valor del potencial matricial de suelo adecuado para cada cultivo y condiciones de desarrollo que optimice la producción y la eficiencia en el uso del agua (EUA) y los nutrientes como demuestran Buttaro et al. (2015) y Létourneau et al. (2015).

El objetivo de este trabajo ha sido comparar la gestión del riego automatizado con tensiómetros, con diferentes consignas de activación, frente a la estimación de la evapotranspiración del cultivo.

\section{2- Materiales y métodos}

El ensayo se ha desarrollado en un invernadero tipo Almería de "raspa y amagado" situado en el Centro IFAPA La Mojonera (Almería), construido sobre un suelo enarenado artificial, característico de los cultivos hortícolas intensivos del sureste peninsular. El suelo presentaba los siguientes perfiles: capa superior de arena de granulometría $1-5 \mathrm{~mm}$ y 6-8 $\mathrm{cm}$ de espesor, capa de tierra de cañada aportada de textura franco-limosa y 20-30 cm de espesor y capa de suelo fértil original de textura franco arenosa, elevada pedregosidad y 20 $30 \mathrm{~cm}$ de espesor. El material vegetal implantado fue un cultivo de calabacín (Cucurbita pepo L.var. Casiopee). El trasplante se realizó el 13 de enero de 2015 a un marco de 1 planta $\mathrm{m}^{-2}$ y el ciclo finalizó el 1 de junio (Figura 1). El riego se aplicó mediante un sistema de riego localizado con emisores autocompensantes y antidrenantes de $3 \mathrm{~L} \mathrm{~h}^{-1}$ y un marco de riego de 2 emisores $\mathrm{m}^{-2}$. El invernadero cuenta con 12 lisímetros de drenaje de $1 \mathrm{~m}^{-2} \mathrm{de}$ superficie, instalados a $50 \mathrm{~cm}$ de profundidad (Figura 3). Se establecieron tres tratamientos de riego. Al tratamiento T1 se le asignó una consigna de potencial matricial de suelo de -10 $\mathrm{kPa}$ y dotación de $1,5 \mathrm{~L} \mathrm{~m}^{-2}$, al T2 una consigna de $-25 \mathrm{kPa}$ y $2 \mathrm{~L} \mathrm{~m}^{-2}$ y al T3 una consigna de $-40 \mathrm{kPa}$ y $3 \mathrm{~L} \mathrm{~m}^{-2}$. La dotación de riego se estableció, considerado la curva de retención de humedad del suelo, al objeto de no alcanzar niveles de humedad en suelo que generasen drenaje. En cada tratamiento se instalaron cuatro tensiómetros electrónicos (Irrometer Co, inc. Riverside, CA, USA) instalados a $15 \mathrm{~cm}$ de profundidad por debajo de la capa de arena (zona radicular) y a $15 \mathrm{~cm}$ de la planta y del gotero. Todos los tensiómetros estaban acoplados a un transductor electrónico que transfería los datos a un equipo de control (Sistema Red Himarcan®) (Figura 2). Cada tratamiento de riego era activado con un único tensiómetro electrónico, el resto se empleaban para registrar medidas del potencial matricial del suelo. 


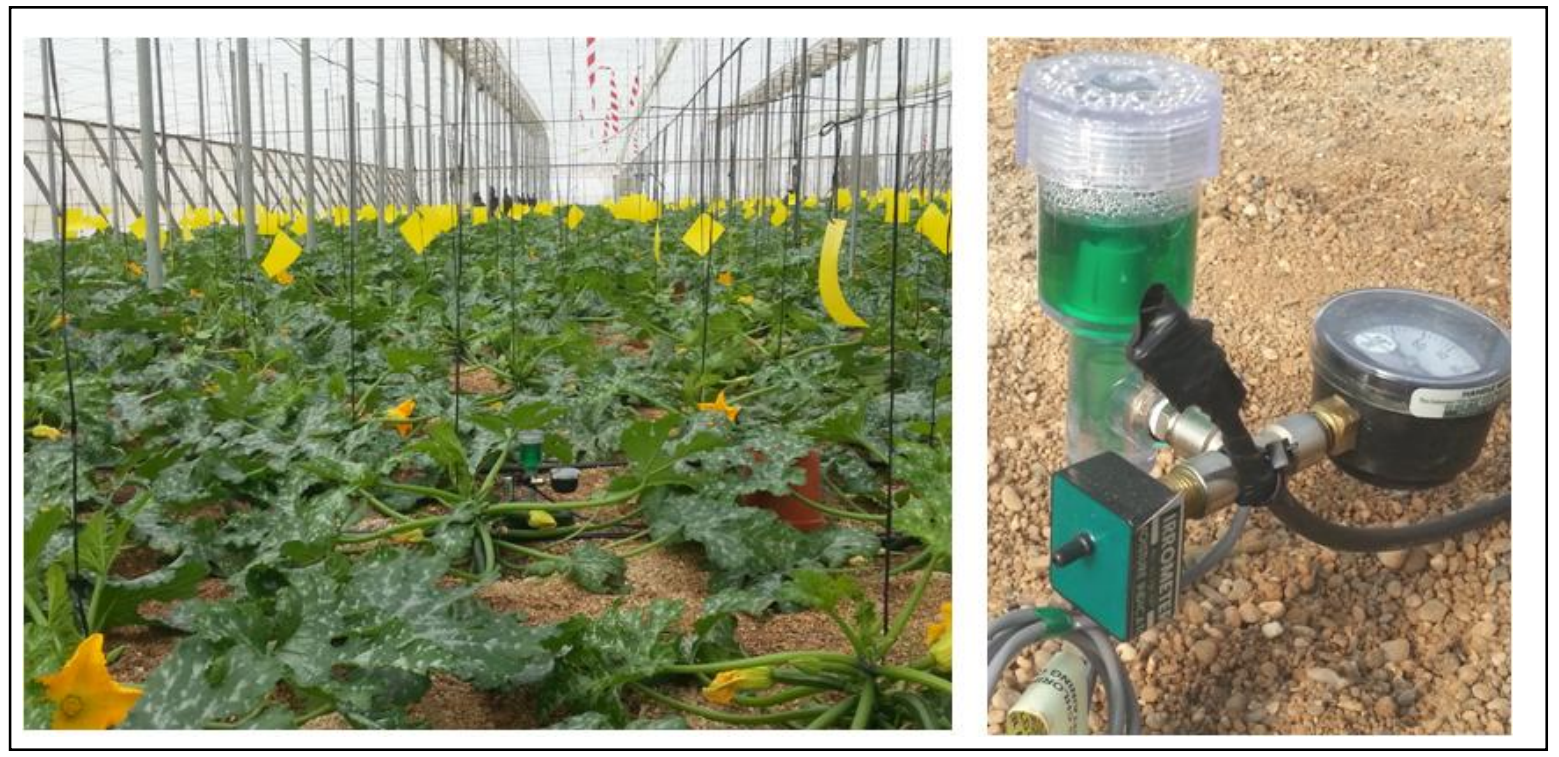

Figuras 1 y 2. Invernadero experimental de calabacín. Tensiómetro electrónico instalado.

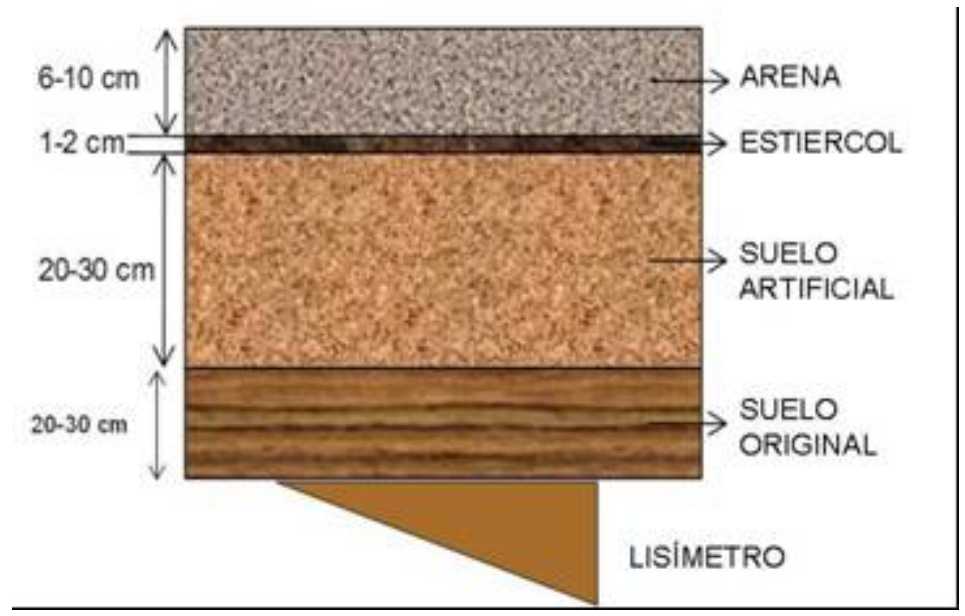

Figura 3. Perfil del suelo y lisímetro

Se realizaron determinaciones del volumen de agua aplicada y volumen de drenaje $\left(\mathrm{L} \mathrm{m} \mathrm{m}^{-2}\right)$, de la producción comercial $\left(\mathrm{kg} \mathrm{m}^{-2}\right)$, del área foliar $\left(\mathrm{cm}^{2}\right)$ y de la biomasa total, expresada en $\mathrm{g} \mathrm{m}^{-2}$ de materia seca. Además, se estimó la evapotranspiración del cultivo $\left(\mathrm{ET}_{\mathrm{c}}\right.$ ) usando el software de riegos PrHo v 2.0 @ 2008 (Fundación Cajamar) para cultivo de calabacín en invernadero. La $\mathrm{ET}_{\mathrm{c}}$ se estima con un modelo que está basado en el modelo propuesto por FAO y que ha sido adaptado para su uso en los cultivos hortícolas en invernadero, de manera que la $\mathrm{ET}_{\mathrm{c}}$ puede ser estimada con precisión a partir de valores medidos de radiación solar exterior y la temperatura dentro de invernadero (Fernández et al. 2001).

La estimación de la $\mathrm{ET}_{\mathrm{c}}$ se ha realizado a partir de datos reales $\left(\mathrm{ET}_{\mathrm{c}-\mathrm{r}}\right)$ y también a partir de datos climáticos medios $\left(\mathrm{ET}_{\mathrm{c}-\mathrm{m}}\right)$. Para la obtención de datos medios, se utilizaron dos series de datos históricas, una para la temperatura interior del invernadero (perteneciente a la Estación Experimental Las Palmerillas, 15 años de registros) y otra para la radiación solar exterior (perteneciente a la estación de La Mojonera de la Red de Información Agroclimática de Andalucía, 10 años de registros).

\section{3- Resultados y discusión}


Los tratamientos han presentado consumos de agua muy diferentes entre sí. El T3 ha recibido la menor cantidad, $272 \mathrm{~L} \mathrm{~m}^{-2}$. En el otro extremo estaba el T1 que ha recibido un $46 \%$ más que el T3. Por su parte, el tratamiento T2 con la consigna intermedia ha generado un consumo de agua de $315 \mathrm{~L} \mathrm{~m}^{-2}$, un $16 \%$ más respecto al T3 (Figura 4 y Tabla 1). La evolución de los consumos de agua a lo largo del ciclo de cultivo muestra curvas paralelas para los tres tratamientos (Figura 4).

Por otro lado, la $\mathrm{ET}_{\mathrm{c}}$ estimada a partir de datos climáticos medios, $\mathrm{ET}_{\mathrm{c}-\mathrm{m}}$, ha arrojado un valor de $264 \mathrm{~L} \mathrm{~m}^{-2}$ que ha sido superado en un $16 \%$ cuando la estimación se ha realizado a partir de datos reales de campaña, $E T_{c-r}=315 \mathrm{~L} \mathrm{~m}^{-2}$. La diferencia entre ambas $E T_{c}$ calculadas son el resultado de una campaña con una radiación y temperaturas diarias superiores a la media en buena parte del ciclo de cultivo. De estos resultados se desprende que la utilización de series climáticas medias para la estimación de la $\mathrm{ET}_{\mathrm{C}}$ es válida, siempre y cuando los valores de evapotranspiración estimados no se alejen de los reales (Bonachela et al. 2009).

La comparación de los consumos de agua de los tratamientos de riego ensayados con la $\mathrm{ET}_{\mathrm{c}-\mathrm{m}}$ y la $\mathrm{ET}_{\mathrm{c}-\mathrm{r}}$ muestra que el tratamiento $\mathrm{T} 3$ presentó un consumo similar al obtenido con la $\mathrm{ET}_{\mathrm{c}-\mathrm{m}}\left(272\right.$ vs. $\left.264 \mathrm{~L} \mathrm{~m}^{-2}\right)$ y el tratamiento $\mathrm{T} 2$ tuvo un consumo igual al calculado mediante la $\mathrm{ET}_{\mathrm{c}-\mathrm{r}}$. Las curvas de evolución de los consumos de agua a lo largo del ciclo de cultivo muestran que estas similitudes entre tratamientos de riego aplicados y las necesidades hídricas calculadas a partir de la $\mathrm{ET}_{\mathrm{c}}$ se producen a lo largo de todo el ciclo de cultivo (Figura 4). La curva del T3 se superpone con la correspondiente a la de la $\mathrm{ET}_{\mathrm{c}-\mathrm{m}}$ y la del T2 se superpone a la de la $\mathrm{ET}_{\mathrm{c}-\mathrm{r}}$ (Figura 4).

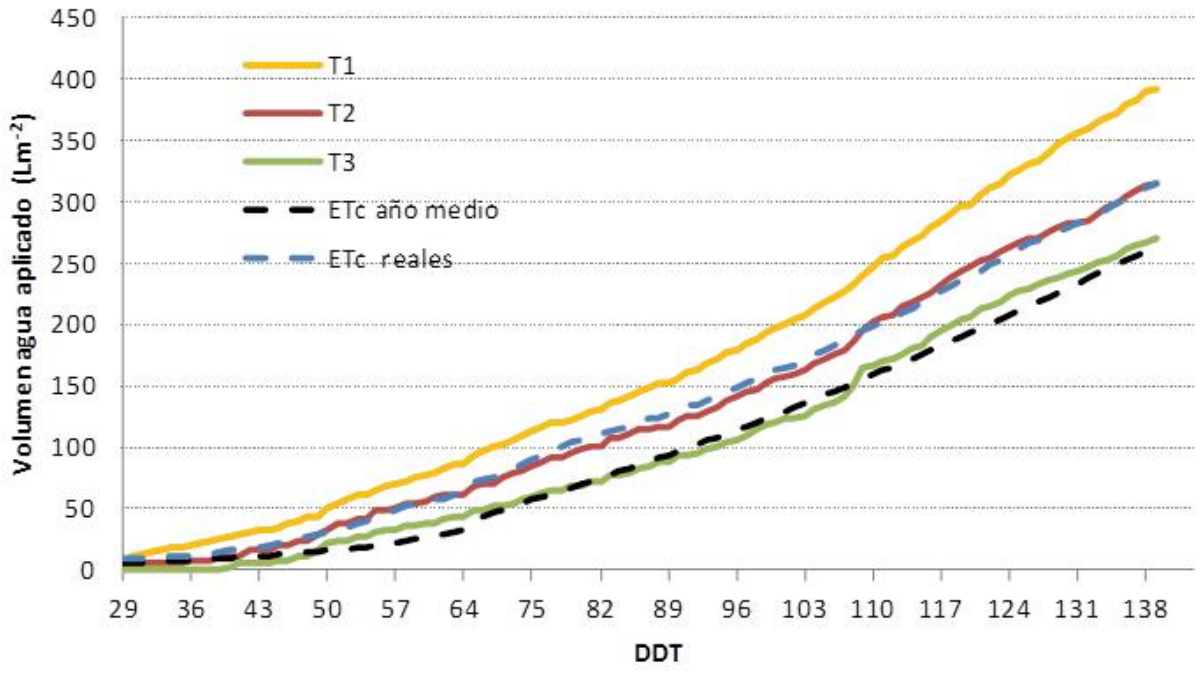

Figura 4. Volúmenes de agua aplicados por tratamiento de riego y volúmenes de agua estimados mediante la $\mathrm{ET}_{\mathrm{c}}$ con datos año medio y la $\mathrm{ET}_{\mathrm{c}}$ con datos reales de la campaña. DDT: días desde trasplante.

Cabe destacar, que durante la campaña de cultivo no se produjo drenaje en ninguno de los tratamientos. Previamente al trasplante y al finalizar el cultivo se realizaron dos riegos de hidratación hasta superar el punto de saturación del suelo recogiendo un volumen significativo de drenaje en todos los lisímetros.

Los tratamientos tuvieron un claro efecto sobre la producción. Los resultados muestran un efecto gradual en función del potencial matricial del suelo, de manera que cuanto menor fue la tensión matricial del suelo menor fue la producción (Tabla 1). El 
tratamiento que activaba el riego a $-40 \mathrm{kPa}$ (T3) redujo la producción en un $23 \%$ con respecto al que activaba el riego a $-10 \mathrm{kPa}(\mathrm{T} 1)$ y en un $14 \%$ con respecto al que activaba el riego a $-25 \mathrm{kPa}$ (T2). Las diferencias entre los tratamientos T1 y T2 fueron del 10\% (Tabla 1).

Tanto las diferencias en producción, como las de consumo de agua entre tratamientos están asociadas al desarrollo de las plantas. La activación del riego a mayor tensión matricial ha derivado en un menor desarrollo de las plantas. La biomasa total en el tratamiento T1 es un 33 \% superior a la obtenida en el tratamiento T3 (Tabla 2).

El tratamiento T3 junto al T2 fueron los que presentaron una mayor eficiencia en el uso del agua (Tabla 1). Teniendo en cuenta que el T2 en términos productivos tuvo una cosecha comercial significativamente superior a la del T3, en las condiciones de este ensayo y que además generó un consumo de agua igual al estimado con la $\mathrm{ET}_{\mathrm{c}}$ a partir de datos climáticos reales, se puede concluir que el T2 con una consigna de $-25 \mathrm{kPa}$ y y una dotación de $2 \mathrm{~L} \mathrm{~m}^{-2}$ fue el que arrojó los mejores resultados.

Tabla 1. Producción comercial, consumo de agua y eficiencia en el uso del agua (EUA) por tratamiento.

\begin{tabular}{cccc}
\hline Tratamiento & $\begin{array}{c}\text { Producción comercial } \\
\left(\mathbf{K g m ~}^{-2}\right)\end{array}$ & $\begin{array}{c}\text { Consumo agua } \\
\left.\mathbf{( L m ~}^{-2}\right)\end{array}$ & $\begin{array}{c}\text { EUA } \\
\left(\mathbf{K g m}^{-3} \mathbf{)}\right.\end{array}$ \\
\hline T1 & $16,6 \mathrm{a}$ & 390 & $42,6 \mathrm{~b}$ \\
T2 & $14,9 \mathrm{~b}$ & 315 & $47,3 \mathrm{a}$ \\
T3 & $12,8 \mathrm{c}$ & 272 & $47,1 \mathrm{a}$
\end{tabular}

Letras diferentes en la misma columna indican diferencias significativas $(p \leq 0.05)$.

Tabla 2. Área foliar, biomasa total (expresada en $\mathrm{g} \mathrm{m}^{-2}$ de materia seca) y porcentaje de la biomasa destinada a fruto por tratamiento

\begin{tabular}{cccc}
\hline Tratamiento & Área foliar $\left(\mathbf{c m}^{2}\right)$ & Biomasa total $\left(\mathbf{g ~ m}^{-2}\right)$ & $\begin{array}{c}\text { Porcentaje de biomasa tota } \\
\text { destinada a fruto }\end{array}$ \\
\hline T1 & $59910 \mathrm{a}$ & $1402 \mathrm{a}$ & $66 \mathrm{a}$ \\
T2 & $46924 \mathrm{~b}$ & $1211 \mathrm{~b}$ & $65 \mathrm{a}$ \\
T3 & $32554 \mathrm{c}$ & $1054 \mathrm{c}$ & $67 \mathrm{a}$ \\
\hline
\end{tabular}

Letras diferentes en la misma columna indican diferencias significativas ( $\leq \leq 0.05)$.

\section{4- Conclusiones y recomendaciones}

El riego automatizado con tensiómetros se presenta como una opción viable teniendo en cuenta su aproximación a las necesidades hídricas del cultivo calculadas mediante la estimación de la $\mathrm{ET}_{\mathrm{c}}$.

Con diferentes consignas de activación se obtienen diferencias productivas y de eficiencia en el uso del agua. Es necesario por tanto establecer consignas adecuadas para cada cultivo.

Las diferencias productivas y de consumo de agua están asociadas al diferente desarrollo de las plantas cuando se trabaja a diferentes tensiones matriciales. 
Del análisis conjunto de la producción comercial, la eficiencia en el uso del agua y la similitud del volumen de agua aplicado con las necesidades hídricas estimadas a partir de la $\mathrm{ET}_{\mathrm{c}}$, el T2 con consigna de activación del riego a $-25 \mathrm{KPa}$ presentó los mejores resultados.

\section{5- Bibliografía}

Bonachela, S., González, A.M. \& Fernández, M.D. (2006). Irrigation scheduling of plastic greenhouse vegetable crops based on historical weather data. Irrigation Sci., 25, 5362.

Buttaro, D., Santamaria, P., Signore, A., Cantore, V., Boari, F., Montesano, F.F. \& Parente, A. (2015). Irrigation management of greenhouse tomato and cucumber using tensiometer: Effects on yield, quality and water use. Agric. Sci. Procedia., 4,440-444.

Fernández, M.D., Orgaz, F., Fereres, E., López, J.C., Céspedes, A., Pérez, J., Bonachela, S. \& Gallardo, M. (2001). Programación del riego de cultivos hortícolas bajo abrigo en el sudeste español. Fundación Cajamar.

Gallardo, M., Fernández, M.D., Thompson, R.B., \& Magán, J.J. (2007). Productividad del agua en cultivos bajo invernadero en la costa mediterránea. Vida Rural, 259, 48- 51.

Jones, H.G. (2004). Irrigation scheduling: advantages and pitfalls of plant-based methods. J. Exp. Bot., 55, 2427-2436.

Létourneau, G., Caron, J., Anderson, L. \& Cormier, J. (2015). Matric potential-based irrigation management of field-grown strawberry: Effects on yield and water use efficiency. Agric. Water Manage., 161,102-113.

Thalheimer, M. (2003). Tensiometer modification for diminishing errors due to the fluctuating inner water column. Soil Sci. Soc. Am. J., 67, 737-739.

Van lersel, M.W., Chappell, M. \& Lea-Cox, J.D. (2013). Sensors for improved efficiency of irrigation in greenhouse and nursery production. HortTechnology, 23, 735-746. 